\title{
A New Energy-efficient Multi-hop Routing Protocol for Heterogeneous Wireless Sensor Networks
}

\author{
Rowayda A. Sadek ${ }^{1}$, Doha M. Abd-alazeem², Mohamed M. Abbassy ${ }^{3}$ \\ Information Technology Department, Faculty of Computers \& Artificial Intelligence, Helwan University, Cairo, Egypt ${ }^{1}$ \\ Information Technology Department, Faculty of Computers \& Artificial Intelligence, Beni-Suef University, Cairo, Egypt ${ }^{2,3}$
}

\begin{abstract}
Energy use of sensor nodes efficiently and extending the lifetime of heterogeneous wireless sensor networks (HWSNs) is a main goal of HWSNs routing optimization methods, and therefore building an energy-efficient routing protocol becomes critical for HWSN performance improvement. They present an energy-efficient routing protocol based on the grey wolf optimizer (GWO) and the Tabu search algorithm (TSA) in this paper. Proposed routing system with primary objectives include clustering and the selection of cluster heads (CH) utilizing GWO with a fitness function based on the residual energy of sensor nodes and the average distance between $\mathrm{CH}$ and sink nodes base station (BS ) due to the mobility of sensors, the quality of service $(\mathrm{QoS})$ parameters such as reliability and energy consumption can be improved by discovering multiple optimized paths for data transmission from $\mathrm{CH}$ to BS and by TSA selecting the optimal route from $\mathrm{CH}$ to $\mathrm{BS}$ based on the forwarding of reliable route packets (FRRPs). The experimental results indicate that the proposed grey wolf optimizer with tabu Search Algorithm (GWO-TSA) can reduce HWSNs energy consumption by $10 \%$ and $20 \%$, increase lifetime by $13 \%$ and $18 \%$, and finally increase throughput by $6 \%$ and $14 \%$ when compared to the genetic algorithm with tabu search algorithm (GA-TSA) and grey wolf optimizer with crow search algorithm (GWO-CSA). When compared to GA-TSA \& GWO-CSA, simulation reveals that the proposed GWO-TSA protocol improves HWSNs performance by minimizing energy consumption, maximizing network lifetime, and boosting network throughput.
\end{abstract}

Keywords-Heterogeneous wireless sensor networks (HWSNs); forwarding of reliable route packets (FRRPs); grey wolf optimizer (GWO); routing optimization; tabu search algorithm (TSA); quality of service (QoS)

\section{INTRODUCTION}

Wireless sensor networks (WSNs) connect the physical and digital worlds via many sensor nodes. Military surveillance, environmental monitoring, medical and healthcare applications all make use of WSNs. Because nodes are mobile, WSNs have a dynamic topology. This frequently results in the battery being unable to be changed or replaced, reducing the network's life, and it is critical to conserve energy and reduce the energy consumption of sensor nodes, as they are critical for communication in Wireless sensor networks. In the event of battery exhaustion, node and link failures may occur, necessitating the immediate suggestion of an alternate route to continue data transmission from the source to the destination, which requires additional energy. Thus, by establishing multiple paths for data communication, the overall efficiency, reliability, and integrity of the wireless sensor network can be increased, and the network's traffic load can be distributed evenly [1].

Sensor nodes in WSNs are made up of wireless transceivers that can collect data from sensors and communicating with one another as in Fig. 1. A self-contained sensor node is a tiny device composed of four major components: sensing, computation, communication, and power. The sensor node's battery capacity is restricted, charging is difficult, and charging may be impossible [2].

There are two types of wireless sensor networks: heterogeneous and homogeneous as in Fig. 2. Heterogeneous wireless sensor networks are composed of sensor nodes with varying capabilities, including varying computational capabilities and sensing ranges, as well as certain sophisticated nodes [3]. The sensor node's primary function is to detect data from the environment and transmit it to the heterogeneous high-level node $\mathrm{CH}$, which has high-level energy or communication capabilities as in Fig. 3. Advanced nodes may be equipped with greater memory and more powerful microprocessors/microcontrollers than cluster member nodes. The primary benefit of HWSNs is that it extends the life of the network, increases data transmission reliability, and reduces latency [4].

By reducing the transmission distance between the $\mathrm{CH}$ and the BS and optimizing the energy consumption of the sensor node, the clustering-based hierarchical routing protocol improves energy efficiency and network lifespan [5]. Clustering splits the region of WSNs sensing into many clusters. Each cluster's $\mathrm{CH}$ is responsible for connecting to other cluster members (CMs), collecting data from them, aggregating it, and transmitting it to the BS as in Fig. 4. As a consequence, how do you optimize your website? The most critical element of clustering-based routing protocols is the process of clustering and choosing $\mathrm{CHs}$ [6].

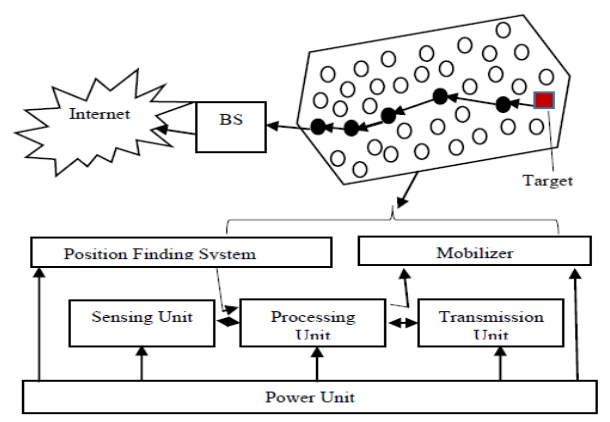

Fig. 1. Sensor Node Structure [16]. 


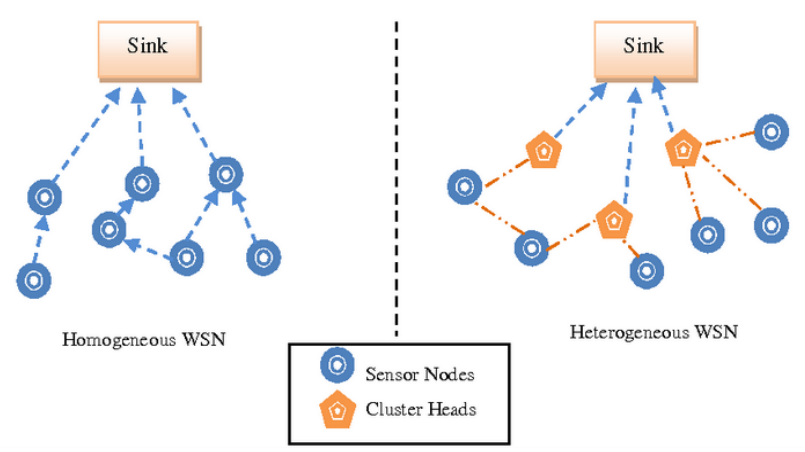

Fig. 2. Homogenous \& Heterogeneous WSN [22].

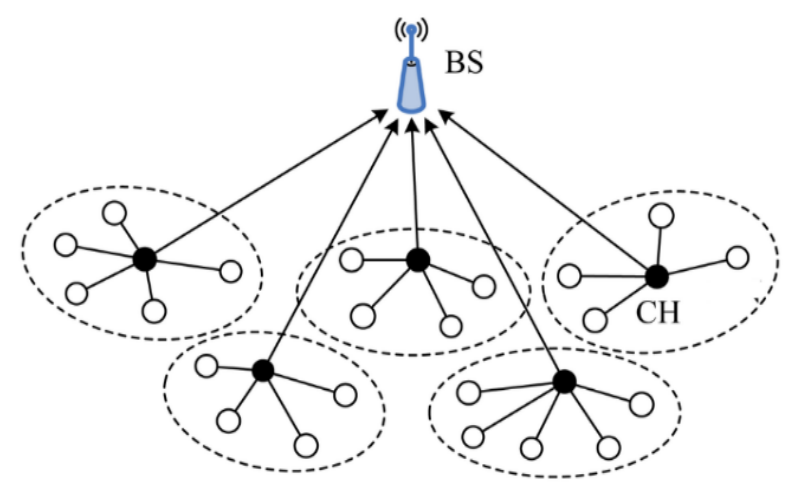

Fig. 3. Heterogeneous WSNs Structure.

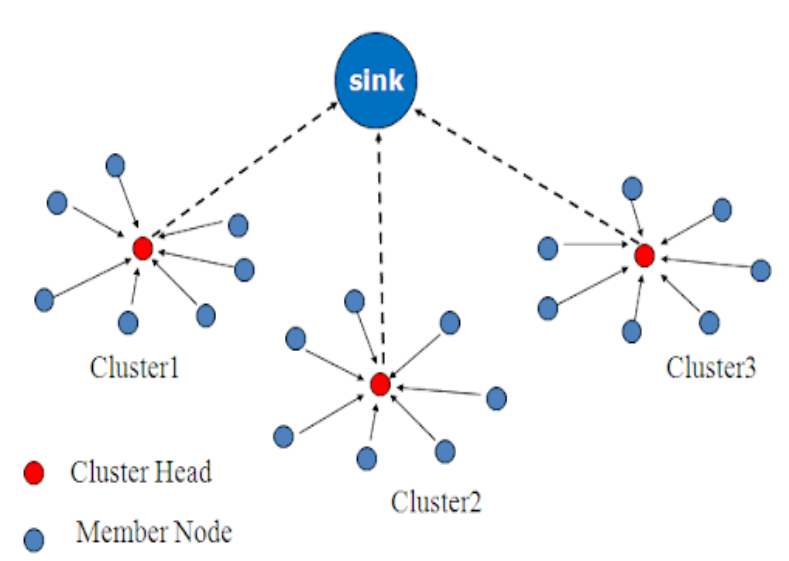

Fig. 4. WSN Clusters Structure [17].

Because a WSN is a self-organizing network, using a static topology increases the routing overhead, resulting in higher congestion and sensor node energy consumption [7]. With $\mathrm{N}$ nodes, the network is said to be enormous in size. Sensors are randomly distributed across a sensing range. Due to the large number of sensor nodes and their tight energy restrictions, as well as the frequent changes in topology, an energy-efficient routing method is critical. This may be achieved via the use of Tabu search, which is based on FRRPs. This search returns the optimal path. FRRPs packets are required for routing choices because they take into account path latency, node energy, and the frequency at which a node acts as a router. In wireless sensor networks, QoS requirements like as latency, throughput, and dependability must be considered [1].

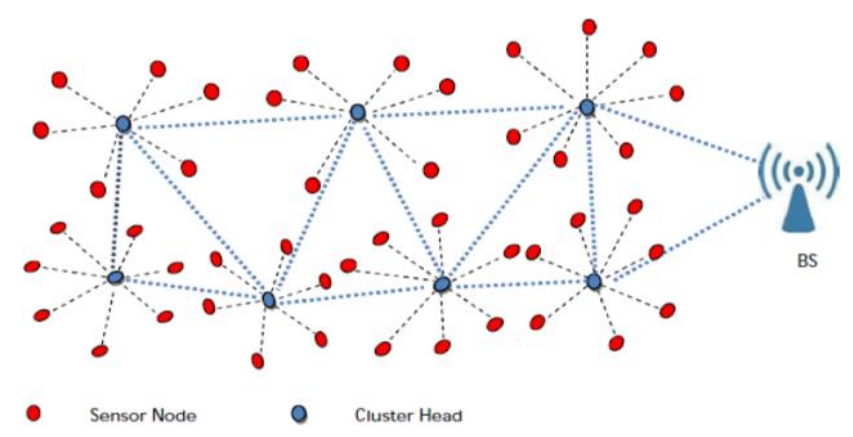

Fig. 5. Hierarchical Routing-Protocol Structure [17].

Routing techniques used in WSNs all have the aim of effectively using the limited resources available to sensor nodes to reduce energy consumption and prolong the network's lifespan [23]. Hierarchical Routing is the most often utilized routing algorithm in wireless sensor networks as in Fig. 5. Its primary objective is to control the energy consumption of sensor nodes effectively to optimize system lifespan and to sustain numerous clusters through multi-hop communication [25] [26]. Each cluster elects a cluster head who is responsible for aggregating data from cluster member nodes, while lowenergy nodes may perform sensing duties. The primary objective is to minimize the number of messages delivered to BS. Clusters are generated using sensor energy storage and proximity to the cluster head [8] [24].

Due to the large number of sensor nodes that are subject to tight energy restrictions and frequent topology changes, the objective is to develop a routing protocol that controls node mobility and optimizes the routing process because of node mobility. The proposed GWO-TSA protocol, which operates on moving nodes in HWSNs, seeks to decrease energy consumption, increase WSN throughput, and prolong the network's life. Since the majority of HWSNs applications need dynamic routing methods, we want to manage the routing Process in such a way that it achieves the critical objective of reducing energy consumption and increasing network lifespan. This goal can be achieved by the use of GWO for clustering and optimize routing process by the selection of $\mathrm{CHs}$ by utilizing GWO with a fitness function based on the residual energy of sensor nodes and the average distance between $\mathrm{CH}$ and BS due to the mobility of sensor nodes and use TSA for selecting optimal route from $\mathrm{CH}$ to $\mathrm{BS}$ based on FRRPs.

The remainder of this paper is arranged as follow. Researcher's related work is described in Section II. Section III introduces routing optimization in WSNs, First introduce GWO, second grey wolf based Optimization for WSNs and finally TSA \& its theoretical analyses. The Proposed Hybrid GWO-TSA protocol and presented in Section IV. Modeling of WSN explained in Section V. Experimental results and discussion are shown in Section VI Finally, Section VII concludes this paper.

\section{RELATED WORK}

Various researchers investigated issues related to the design of a routing protocol for a dynamic WSNs topology and its routing issues in order to send data from source nodes to the BS using a variety of techniques with the primary goal of 
achieving routing optimization in WSNs by reducing sensor nodes' energy consumption, extending the network's lifetime, and increasing network performance.

A cluster head-based routing hierarchy method proposed for increasing the energy efficiency of WSNs by splitting the routing responsibility between a cluster head and a cooperative vice cluster head. The approach is similar to that of a TX/RX load balancing issue, except that the vice cluster head selection criteria are determined by the $\mathrm{CH}$, resulting in a transparent temporary responsibility transfer procedure for all normal nodes in a cluster after the death of the cluster head. When compared to current vice cluster head-based techniques, the suggested system has the benefit of not needing time for each round's advertising phase, since the authority transfer procedure from a $\mathrm{CH}$ to its vice $\mathrm{CH}(\mathrm{VCH})$ does not interrupt WSN operation. According to simulation data, attaching a vice $\mathrm{CH}(\mathrm{VCH})$ node to share routing duties with a $\mathrm{CH}$ improves load balance across the WSN, thus prolonging its lifespan [9].

MLHP (Multi-level Hybrid Clustering Routing Protocol) was suggested as a technique for optimizing routing in WSNs using the GWO. To begin, the BS is critical in the $\mathrm{CH}$ selection process, and the second routing step utilizes GWO to transmit data to the BS. To save energy, nodes choose the most efficient route to the BS. \& a third-stage clustering method based on a cost/fitness function calculated from the node's residual energy and the number of its neighbors. MLHP beat the other algorithms in terms of network lifespan, stability period, and residual energy [10].

Dynamic Cluster Formation Method was developed to prolong the network's lifespan. Based on coverage and connectivity, a WSNs is split into many clusters. The coverage range of a network is first confirmed by all nodes. This is done by broadcasting a message to all of the nodes in its vicinity. The nodes within the sensing range transmit an update message to that node. To decide which $\mathrm{CH}$ to utilize, the residual energy and delay time are used. To begin, each node sends a broadcast message to all other nodes. As a consequence, the node count is defined as the number of broadcast messages that a particular node receives. The delay time is calculated based on the number of nodes. The cluster head is the node with the lowest delay time and the most energy left. Clusters are generated by the cluster head. During data transmission, the energy of all nodes is drained. A threshold value is assigned to the energy of a node. When the energy of the $\mathrm{CH}$ falls below a predefined threshold, another node is selected as the cluster head based on the remaining energy and delay time. Therefore, clusters are generated dynamically by moving the cluster heads. Therefore, energy consumption is balanced, extending the network's life [2].

FIGWO (Fitness value-based Improved GWO) is an Energy-Efficient protocol that was implemented based on an improved GWO that utilizes a fitness Function to improve the selection of the optimal solution in GWO, resulting in a more balanced cluster structure and better distribution of $\mathrm{CHs}$. It enhanced the process of choosing $\mathrm{CHs}$ by calculating a fitness value, which ensures that the cluster node nearest to the BS and with the most energy is more likely to be selected as a $\mathrm{CH}$. When a new $\mathrm{CH}$ is chosen, the transmission distance between each node and the BS is also changed. This reduces both the transmission distance between $\mathrm{CHs}$ and BS and the energy consumption of sensor nodes. As a consequence, the lifespan of the network may be prolonged. According to simulation findings, the suggested algorithm's performance was a fitness value that improved the discovery of the optimum solution in GWO, which ensures a more balanced cluster structure and a better distribution of CHs. When compared to other algorithms, this method consumes less energy, maintains network stability, and increases network throughput (amount of data received by BS) [6].

Routing Protocol Framework was developed with the following primary objectives: construct dynamic topology using a genetic algorithm with mutation operator, determine the optimal route for data transmission from $\mathrm{CH}$ to $\mathrm{BS}$ using the Tabu search approach, and improve service quality characteristics such as reliability and energy constraints by discovering various optimum paths. The suggested approach starts by clustering normal sensor nodes. Each cluster is given a $\mathrm{CH}$ using a genetic method. In GA, the fitness function is defined only in terms of energy dissipation. If the sensor nodes' remaining energy is more than the average energy of all live (active) nodes, the BS activates them to maximize energy efficiency. Then, using Tabu search, connect the member nodes to the cluster heads for data transmission. Due to the proposed protocol's optimal route selection technique, simulation results indicate that it may enhance service quality [1].

Routing technique based on the TSA and a fuzzy inference system (FIS) was proposed to enhance network stability, energy consumption, and packet delivery ratio (PDR). To get excellent results in WSN, this technique utilized the TSA method for neighborhood solution selection and next-hop selection. It then applied FIS to the TSA solutions generated, resulting in a route that complied with FIS requirements for data transmission between $\mathrm{CH}$ and BS. The FIS is computed by dividing $\mathrm{CH}$ by $\mathrm{BS}$. The simulation findings indicate that the proposed protocol may enhance service quality by using an optimum route selection method for data transmission from $\mathrm{CH}$ to BS [11].

Optimum cluster head selection method based on a combination of GWO and CSA was created for extending the lifespan of WSNs by reducing the latency in data transmission from $\mathrm{CH}$ to $\mathrm{BS}$, the distance between nodes and $\mathrm{BS}$, and optimizing energy consumption. To address the issue of early convergence, which prevents it from traversing the search space efficiently, CSA is combined with the GWO method. To select $\mathrm{CH}$ efficiently, this hybridization of GWO and CSA algorithms preserves the trade-off between exploitation and exploration degrees in the search space throughout the clustering process. The proposed hybridization of the GWO and CSA protocols demonstrated that by lowering node energy consumption and balancing active and dead nodes, the lifespan of a WSN may be extended [12]. 


\section{ROUTING OPTIMIZATION IN WSNS}

\section{A. Grey Wolf Optimizer}

GWO is an intelligent algorithm that simulates the hierarchy and hunting process of wild wolf packs. Wolves have a four-tiered social structure. As shown in Fig. 6, alpha $(\alpha)$ wolves, beta $(\beta)$ wolves, delta $(\delta)$ wolves, and omega $(\omega)$ wolves are ranked from top to bottom [18].

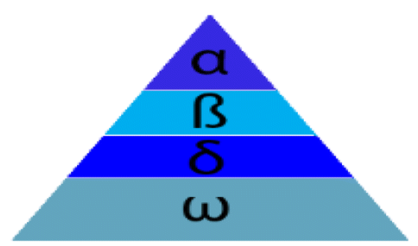

Fig. 6. Hierarchy of GWO [13].

Hunting behavior of the wolf is split into three phases: tracking and approaching prey, hunting, and encircling prey, and attacking prey. The GWO algorithm is led in its hunting (optimization) by, and. wolf is iteratively enhanced via the use of, and. [13] develops the following mathematical model of GWO:

1) Encircling prey: To mathematically simulate the wolf's encircling technique, GWO analyses two points in ndimensional space and changes the location of one of the points in response to the position of the other. As in [13] and [14], the following formulae have been suggested to simulate this.

$\mathrm{D}=|\mathrm{C} * \mathrm{XP}(\mathrm{t})-\mathrm{X}(\mathrm{t})|$

$\mathrm{X}(\mathrm{t}+1)=\mathrm{XP}(\mathrm{t})-\mathrm{A} * \mathrm{D}$

Where $\mathrm{D}$ he distance between the source node (grey wolf) and Destination BS, $t$ is the current iteration, $X_{\mathrm{p}}$ and $X$ indicate the position vector of the prey (BS) and a grey wolf respectively, $A$ and $C$ are coefficient vectors which are calculated as follow as in [13] [14].

$\mathrm{A}=2 \mathrm{a} * \mathrm{r} 1-\mathrm{a}$

$\mathrm{C}=2 * \mathrm{r} 2$

where components of a are decreased linearly from 2 to 0 with iteration, $\mathrm{r} 1$ and $\mathrm{r} 2$ are randomly generated vectors in $[0$, $1]$.

2) Huntting: Gray wolves can identify and approaching prey (BS). Alpha is primarily responsible for hunting, sleeping, and waking up. Beta and delta wolves may also take part in decision-making or other tasks on occasion. Grey wolves' hunting technique may be mathematically approximated using alpha, beta, and delta solutions to approximate the prey (BS) location. Each wolf may update its location using the equations described in [13] and [14].

$\mathrm{D} \alpha=|\mathrm{C} 1 * \mathrm{X} \alpha-\mathrm{X}|$
$\mathrm{D} \beta=\mathrm{C} 2 * \mathrm{X} \beta-\mathrm{X}$
$\mathrm{D} \delta=|\mathrm{C} 3 * \mathrm{X} \delta-\mathrm{X}|$
$\mathrm{X} 1=\mathrm{X} \alpha-\mathrm{A} 1 * \mathrm{D} \alpha$

$\mathrm{X} 2=\mathrm{X} \beta-\mathrm{A} 2 * \mathrm{D} \beta$

$\mathrm{X} 3=\mathrm{X} \delta-\mathrm{A} 3 * \mathrm{D} \delta$

$\mathrm{X}(\mathrm{t}+1)=[\mathrm{X} 1(\mathrm{t})+\mathrm{X} 2(\mathrm{t})+\mathrm{X} 3(\mathrm{t})] / 3$

3) Exploreation \& Explotation in attacking prey: GWO reduced the value of a to mathematically simulate approaching the prey (BS). The fluctuation range of A is likewise reduced by $a$, where $A$ is a random variable in the range $[a, a]$, with a decreasing from 2 to 0 over repetitions. When $|\mathrm{A}| 1$, candidate solutions tend to converge near the prey (BS) and diverge from it when $|\mathrm{A}|>1$. The adaptive values of a and $\mathrm{A}$ determine the shift between exploration and exploitation. When $|\mathrm{A}|>1$, the focus is on exploration, while when $|\mathrm{A}| 1$, the focus is on extraction.

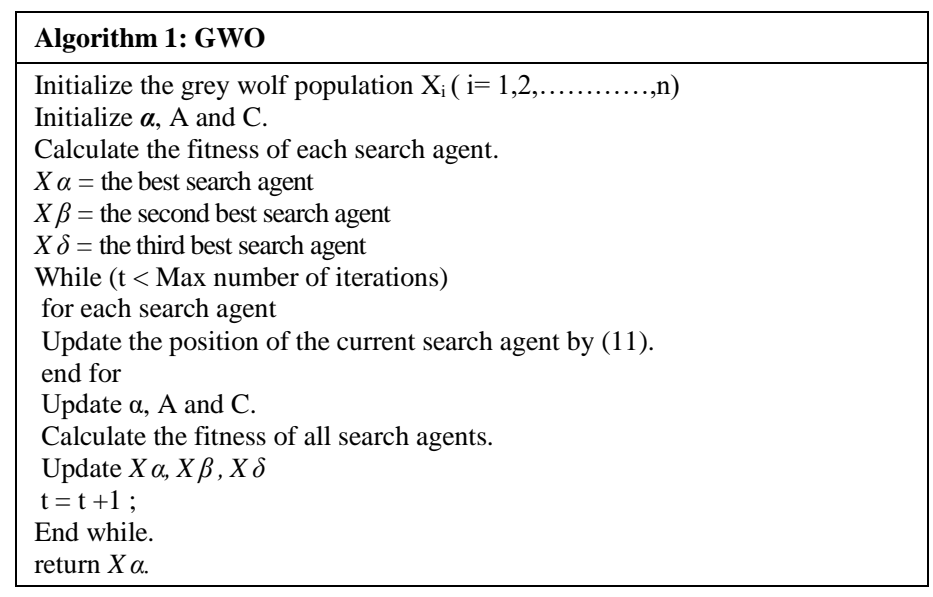

4) Optimization for WSNs: Because the communication process utilizes the majority of the energy in a wireless sensor network, power consumption may be reasonably lowered by carefully choosing the routing technology. The whole network life cycle is reduced as a result of improper data packet routing, and nodes responsible for forwarding data packets play a significant role in routing. An appropriate cluster head must be chosen in light of these issues. This is accomplished by using the remaining energy and the suggested protocol's distance to the BS. To minimize iterations, GWO is employed, in which a layered design splits the whole region into four levels, each with several duties. The first layer's nodes are referred to as leader nodes. When the number surpasses two, game theory may be used to elect the head. This technique results in substantial energy savings, extending the life of the whole network [19].

Cluster-based selection is used to determine which cluster heads are on the first layer and closest to the base station. If layer 1 has several nodes, the nodes' remaining energy is utilized to make a choice. GWO technique adheres to the Gray Wolf's leadership hierarchy. It has four distinct subspecies of grey wolves: alpha $(\alpha)$, beta $(\beta)$, delta $(\delta)$, and omega $(\mathrm{w})$, each of which performs a specific function. The following actions must be taken to establish this leadership hierarchy in a wireless sensor network. Utilize hierarchical and cluster-based 
architectures. Sensor nodes are placed in various levels based on their distance from the base station, with each layer consisting of several sensor nodes [15].

Each layer is separated by $\mathrm{R}$, the first layer is separated by $\mathrm{R}$, and the second layer is separated by $2 \mathrm{R}$. The network is similarly divided into four layers: layer 1 , layer 2 , layer 3 , and layer 4. Clustering is determined by the sensor nodes' density, and the cluster's transmission radius will be $2 \mathrm{R}$. The cluster's chief (or)The leader is elected from the sensor nodes in layer 1, co-leaders are chosen from the nodes in layer 2, elders are elected from the nodes in layer 3, and members are elected from the nodes in layer 4, as shown in Fig. 7.

\section{B. Tabu Search Algorithm}

Tabu search is a meta-heuristic search method that avoids looping back to previously used routes by storing information about adjacent nodes in short-term memory called Tabu lists. Tabu lists include information about neighboring nodes' energy levels, their IDs, and status. To prevent loops, Tabu search utilizes memory based on recency to avoid some re-instancing within a certain time (this is done through a tabu list) [21]. As a result, the Tabu list stores the active routes that are accessible at each energy level. This kind of information is represented in the form of dynamic data packets termed FRRP, which are utilized during data transmission to determine the optimal way from the source to the sink among the many possible routes as in Fig. 8.

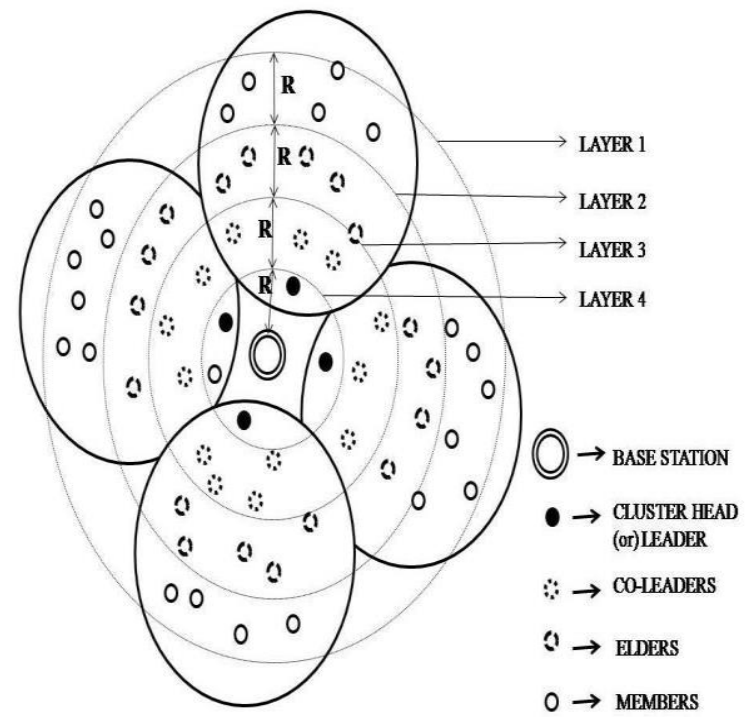

Fig. 7. A Grey-Wolf Optimization Approach [20].

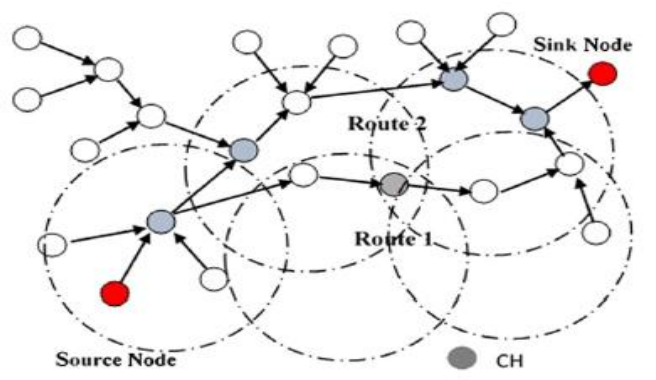

Fig. 8. Optimal Path Selection from Multiple Available Paths [1].

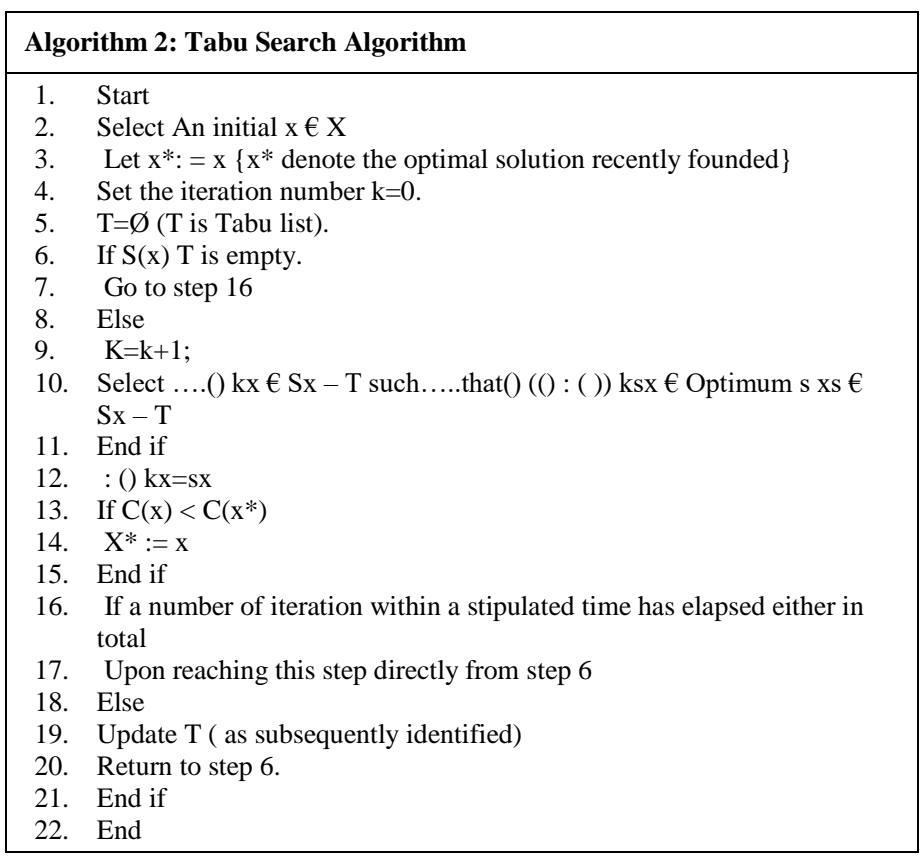

\section{PROPOSED GWO-TSA PROTOCOL}

Proposed GWO-TSA Protocol combines the GWO method with a fitness function for clustering and a procedure for selecting $\mathrm{CHs}$ based on sensor node residual energy and the average distance between the $\mathrm{CH}$ and BS. Then, using TSA with FRRPs for optimum route selection from $\mathrm{CH}$ to $\mathrm{BS}$, enhance QoS factors such as reliability and energy restrictions in WSN. The proposed GWO-TSA aims to improve WSN performance by reducing sensor energy consumption, extending the network's lifespan, and increasing network throughput.

\section{A. Fitness Function}

The proposed GWO-TSA identified $\mathrm{CHs}$ based on the GWO and computed their fitness values using Fitness Function (12), where the fitness value of a node is determined by its distance from the BS and residual energy.

$\mathrm{F}=\mathrm{w} *(\mathrm{TDN}-\mathrm{BS}-\mathrm{DCH}-\mathrm{N})+(1-\mathrm{w}) *(\mathrm{Et}-\mathrm{Er})$

Where the $\mathrm{w}$ coefficient in the fitness function shows the contribution of the residual energy and distance parameters?

$\mathrm{TD}_{\mathrm{N}-\mathrm{BS}}$ - represents the total distance from all nodes to BS.

$\mathrm{D}_{\mathrm{CH}-\mathrm{BS}}$ - represents a distance from $\mathrm{CH}$ to its related Nodes in its cluster.

$\mathrm{E}_{\mathrm{t}}$ - represents the total energy of nodes.

$\mathrm{E}_{\mathrm{r}}$ - represents residual energy of nodes.

\section{B. Steps of the Proposed GWO-TSA in a Dynamically Deployed WSN for Optimal Route Selection for Data Transmission from $\mathrm{CH}$ To BS in Fig. 9}

1) Setup Initialization of the HWSN and deployment of sensor nodes.

2) Clustering using GWO with a fitness function based on the node's distance to the $\mathrm{BS}$ and residual energy for $\mathrm{CHs}$ 
selection (grey wolves, alpha $(\alpha)$, beta $(\beta)$, and delta $(\delta)$ ) three optimum CHs.

3) Initiate TSA by generating FRRPs from (grey wolves, alpha $(\alpha)$, beta $(\beta)$, and delta $(\delta))$ three optimum CHs energy values for each sensor node and its distance from the BS and inserting them into the Tabu list.

4) Using the TSA method on the values in the Tabu list, choose the optimum route for data transfer from $\mathrm{CH}$ to $\mathrm{BS}$ from the possible routes in the Tabu list.

5) Remove FRRP from the Tabu list if it transmits from $\mathrm{CH}$ to $\mathrm{BS}$.

6) Conduct an energy dissipation analysis and update the Tabu list.

7) Repeat steps 2-6 above until all data has been sent from $\mathrm{CH}$ to $\mathrm{BS}$.

\section{Improving QoS Parameters by using Proposed GWO-TSA}

The GWO-TSA proposal is primarily concerned with network quality metrics like as network lifespan, node energy consumption, and network throughput as shown above in Fig. 10.

Dynamic networks, node deployment, network scalability, and network reliability are all linked issues. These issues have to be resolved in order to improve QoS in areas like as dependability, energy consumption, throughput, data loss, and network lifespan.
1) Node deployment: Instead of randomly dispersed nodes, GWO is utilized to deploy sensor nodes depending on their remaining energy and distance to the base station.

2) Formation of Dynamic Clusters: Network nodes this needed more time and energy for the findings to be moved into dynamic clusters and the $\mathrm{CH}$ selection process. This is enhanced using GWO with a Fitness Function to choose CHs based on two primary criteria (energy and distance to BS), which significantly lowers the amount of time and energy spent on the clustering process.

3) Network Scalability: The proposed GWO-TSA performance is unaffected by the number of sensors nodes but is influenced by the node's energy and distance to the base station.

4) Network Reliability: TSA identifies several optimal routes for data transmission from $\mathrm{CH}$ to $\mathrm{BS}$ to increase data transmission reliability; rather of using a single path, many paths may be utilized concurrently.

5) Network Throughput: Data transfer from $\mathrm{CH}$ to $\mathrm{BS}$ was improved by delivering data through several optimal routes identified by TSA. This allows load balancing to concentrate on nodes that influence the network's lifespan rather than on the whole network.

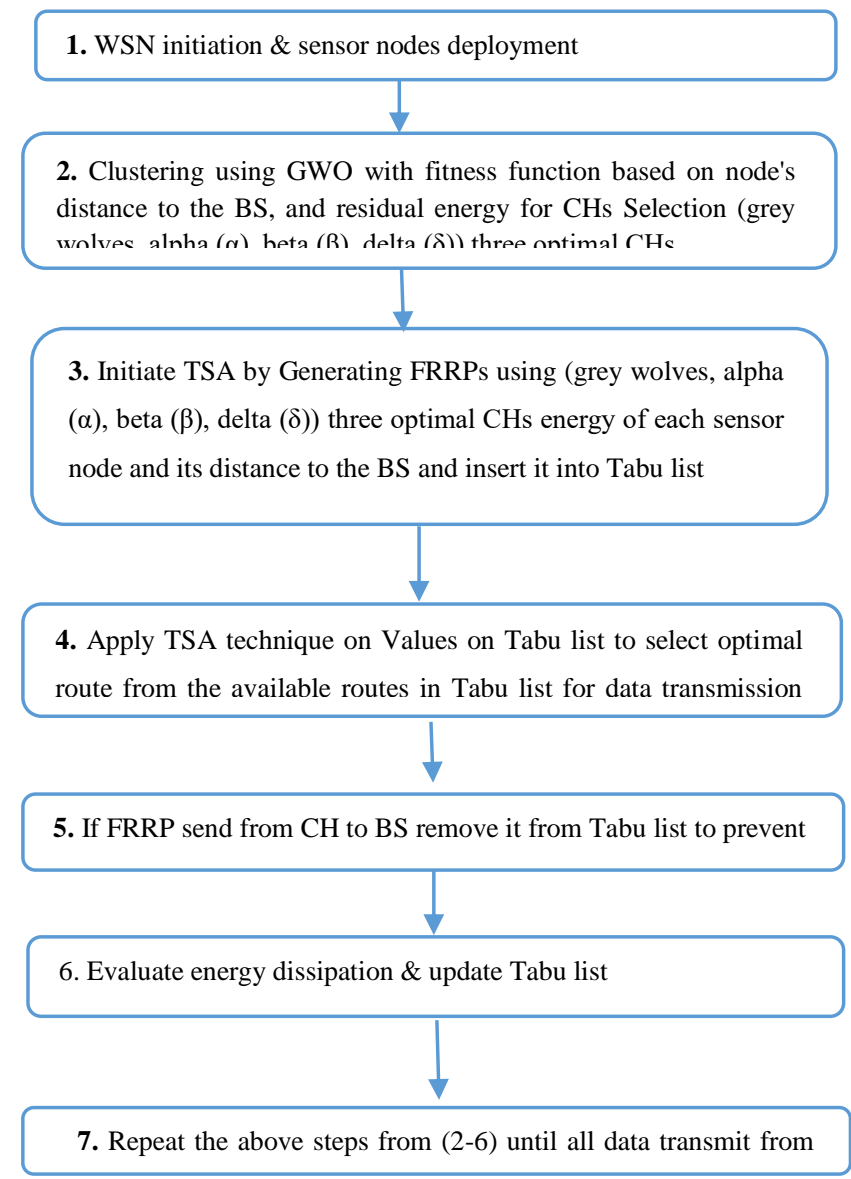

Fig. 9. Proposed GWO-TSA Main Steps. 


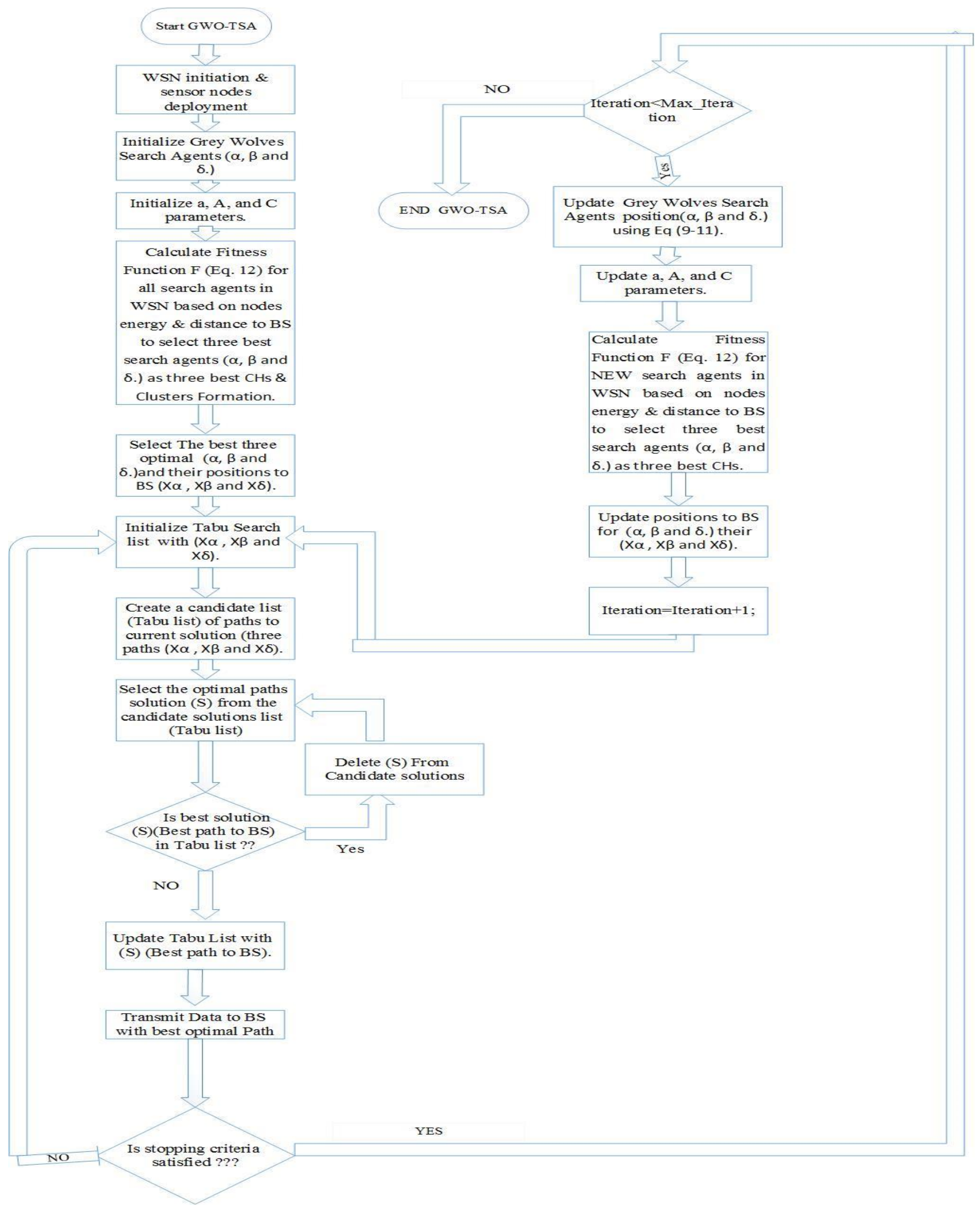

Fig. 10. Proposed GWO-TSA Protocol Flowchart. 


\section{MODELING OF WSNS}

\section{A. Energy Model}

When transmitting an L-bit long data packet across the distance d, the energy model is utilized, as shown in [2]. Specifically, the amount of energy needed is:

$\mathrm{E}_{\mathrm{TX}}(l, d)=l \times \mathrm{E}_{\text {elec }}+l \times \varepsilon_{f_{s}} \times d^{2}$, if $d<d_{0}$

$l \times \mathrm{E}_{\text {elec }}+l \times \varepsilon_{m p} \times d^{4}$, if $d>d_{0}$

Where $\mathrm{E}_{T X}$ indicates the energy transferred and $\mathrm{E}_{\text {elec }}$ indicates the energy used per bit by the transmitter or receiver circuit. The values for $f s$ and $m p$ are dependent on the transmitter amplifier type. The open space model is utilized if the distance between the transmitter and receiver is less than a threshold $\mathrm{d} 0$; otherwise, the multi-path model is used. $d_{0}$ is often computed as follows:

$d_{0}=\varepsilon_{f s} / \varepsilon_{m p}$

\section{B. Simulation Model}

\section{1) Assumptions made for this routing:}

a) The sink (Base Station) is located inside the sensor field.

\section{b) Heterogeneous WSN.}

c) After deployment sensor nodes are unattended. So that recharging or changing of battery is not possible.

d) Links are asymmetric because of nodes mobility.

e) Mobility of the node is controllable and predictable.

Proposed GWO-TSA is done in MATLAB 2017b with 16GB RAM, 1TB HDD, and an i7 CPU. The GWO-TSA network and energy models were built in MATLAB to assess the algorithm's performance.

The following metrics used performance evaluation:

1) Network lifetime.

2) Network Throughput.

3) Network Residual /Remaining energy.

The proposed GWO-TSA aims to improve WSN by reducing sensor energy consumption, extending network lifespan, and increasing network throughput.

\section{RESULTS AND DISCUSSION}

GWO-TSA algorithm is compared to GA-TSA) and GWOCSA algorithms when the identical WSN simulation parameters Table I are being used.

Fig. 11 show Wireless sensor network simulation area as $(200 * 200) \mathrm{m} 2$, where sensor nodes deployment and BS are in the center of the sensing area while Fig. 12 Heterogeneous WSN nodes.

\section{A. Network Lifetime}

It is used to determine the WSN's stability period; it is one of the most critical metric parameters in WSNs. We depict it as a series of alive $\&$ dead nodes with varying round counts.

Fig. 13 show number of alive sensor nodes for three comparative procedures with a varied number of rounds $(0-$
2800 rounds). Initially, when the number of rounds grew from 0 to 2800 number of alive nodes decreased. Proposed GWOTSA, as shown in Fig. 13 maintains nodes alive for a greater number of cycles than the GA-TSA and GWO-CSA. Nodes remain viable for about 2780 rounds in the planned GWOTSA, but only for approximately 2630 and 2260 rounds in the GA-TSA and GWO-CSA, respectively.

TABLE I. SiMULATION PARAMETERS

\begin{tabular}{|l|l|}
\hline Simulation parameters & Value \\
\hline Number of sensor nodes & 100 \\
\hline Sensing Area & $(200 * 200)$ \\
\hline Position of the sink node & $(100 * 100)$ \\
\hline The initial energy of the sensor nodes & $0.5 \mathrm{~J}$ \\
\hline Control packet size & 2000 \\
\hline Data packet size & 4096 \\
\hline Data Aggregation Energy & $\mathrm{E}_{\mathrm{DA}}=50 \mathrm{~nJ} / \mathrm{bit}$ \\
\hline Transmitter/receiver energy & Eelec $=50 \mathrm{~nJ} / \mathrm{bit}$ \\
\hline Transmitter Amplifier(free space) & $\varepsilon_{\mathrm{fs}}=10 \mathrm{~nJ} /\left(\mathrm{bit} . \mathrm{m}^{2}\right)$ \\
\hline Transmitter Amplifier (multi-path space) & $\varepsilon_{\mathrm{mp}}=0.0013 \mathrm{pJ} /\left(\mathrm{bit}^{4} \mathrm{~m}^{4}\right)$ \\
\hline Maximum node speed & $10 \mathrm{~m} / \mathrm{s}$ \\
\hline W & 0.3 \\
\hline
\end{tabular}

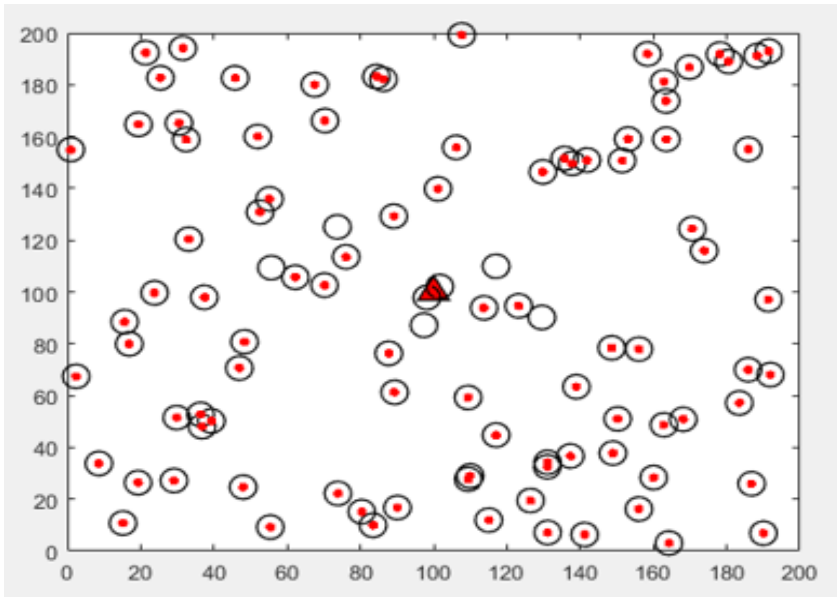

Fig. 11. Wireless Sensor Network Simulation Area.

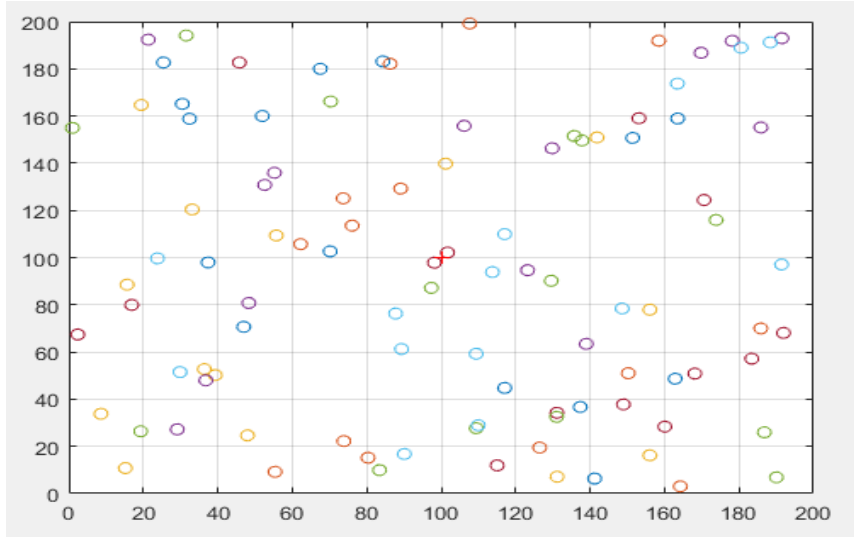

Fig. 12. Heterogenous WSN Nodes. 


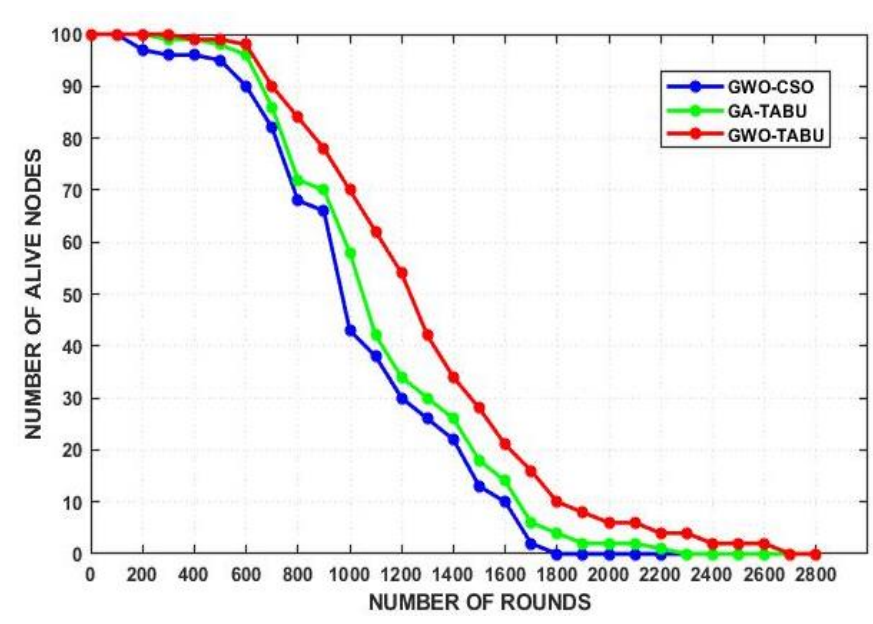

Fig. 13. Number of Alive Nodes in a different Number of Rounds.

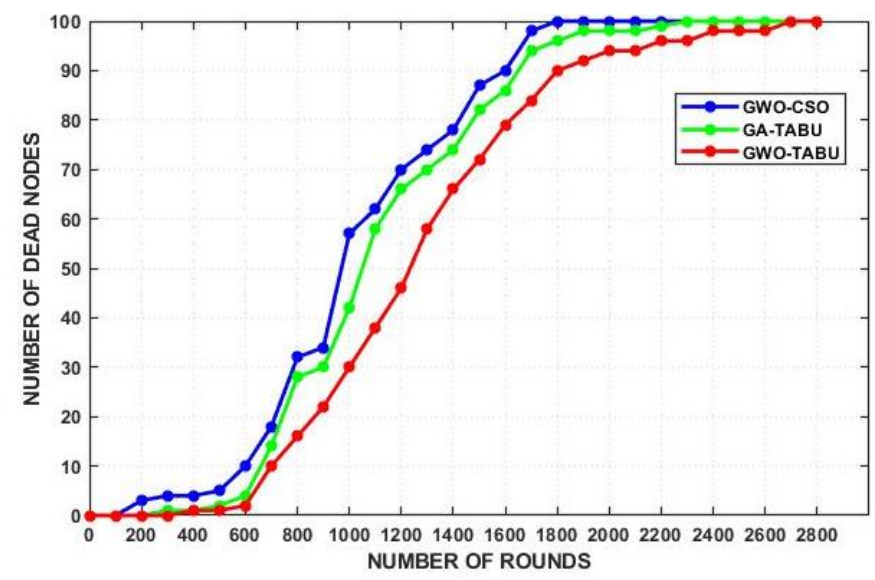

Fig. 14. Number of Dead nodes in a different Number of Rounds.

Fig. 14 show number of dead sensor nodes for three comparative procedures with a varied number of rounds $(0-$ 2800 rounds). Initially, the number of dead nodes increased as the number of rounds increased from 0 to 2800 , as sensor nodes lost energy and their batteries became difficult to recharge. Fig. 14 shows that nodes died approximately at 2780 rounds in the proposed GWO-TSA, while they died at 2630 and 2260 rounds in the GA-TSA and GWO-CSA, respectively. Finally, the Dead \& Alive nodes findings indicate that the proposed GWO-TSA reduces node energy consumption, which results in nodes being alive longer, which increases network lifespan, one of the most critical aspects in WSN performance.

Fig. 15 shows WSN lifetime with a different number of sensor nodes Start with 100 sensors until 1000 sensors node to show network lifetime for three compared protocols. Fig. 15 show that the proposed GWO-TSA enhances network lifetime by improving node energy consumption that leads to nodes alive more times. GWO-TSA improves network lifetime percentage by $13 \%$ \& $18 \%$ over the compared protocols GATSA \& GWO-CSA respectively.

\section{B. Network Throughput}

It's defined as the Number of packets received by BS from $\mathrm{CHs}$, It's another necessary facto in WSN Routing optimization.

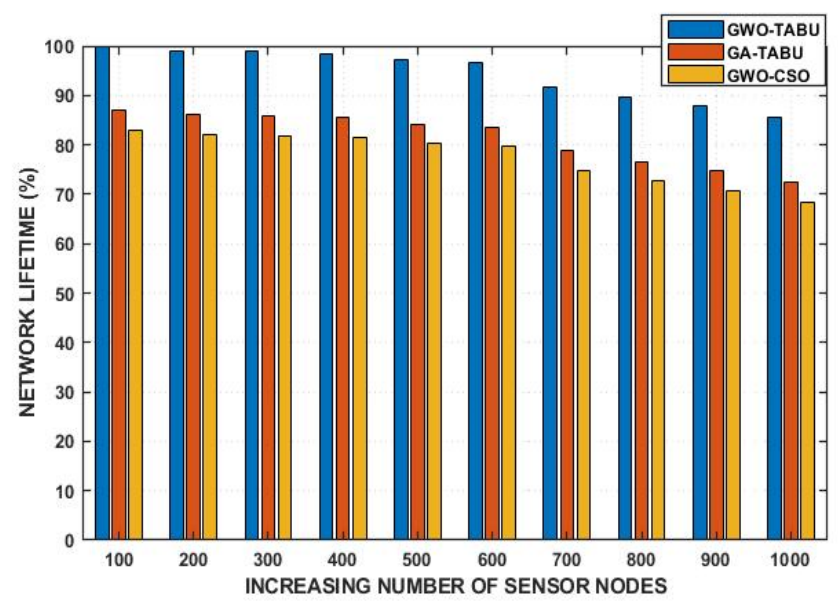

Fig. 15. Network Lifetime of a different Number of Sensor Nodes.

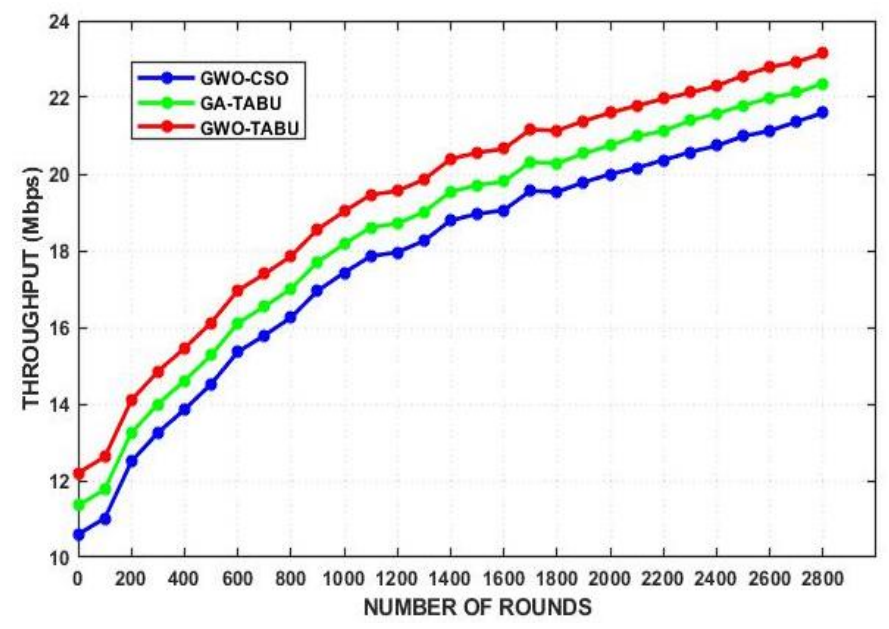

Fig. 16. Network Throughput with a different Number of Rounds.

Fig. 16 show Average of WSN Throughput with a different number of rounds from (0-2800 rounds) for three compared protocols. Fig. 16 shows that the proposed GWO-TSA increases Network Throughput over the compared GA-TSA \&GWO-CSA protocols.

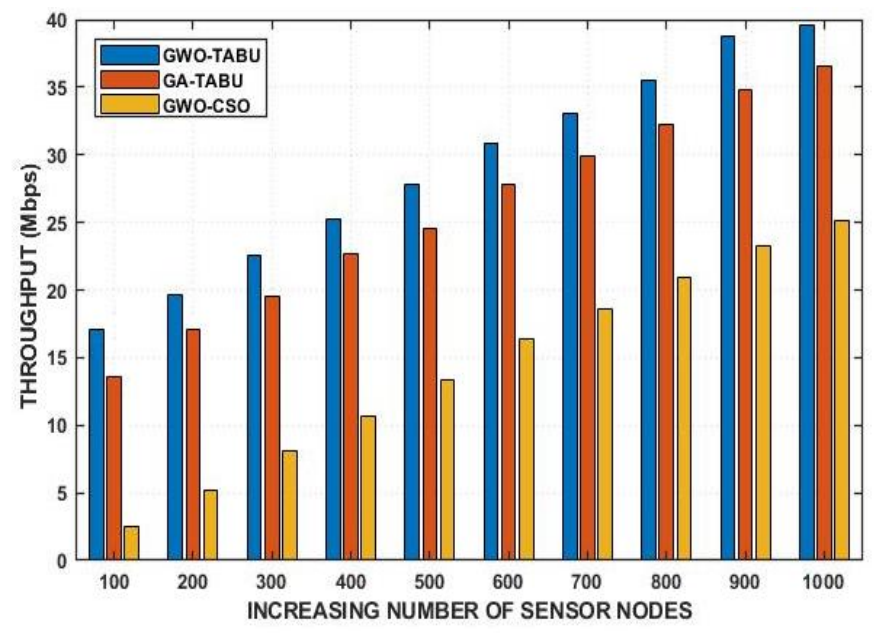

Fig. 17. Network throughput of a different Number of Sensor Nodes. 
Fig. 17 show average of WSN throughput with a different number of sensors nodes Start with 100 sensors until 1000 sensors node to show network throughput for three compared protocols. Fig. 17, show that the proposed GWO-TSA improved network throughput percentage by $6 \% \& 14 \%$ over GA-TSA \& GWO-CSA respectively, this means GWO-TSA increases the number of bits sends by CHs to BS.GWO-TSA using GWO \& Tabu search techniques for clustering \& Cluster heads selection for transmitting aggregated data from cluster nodes to BS by $\mathrm{CHs}$ to save energy for data transmission this lead to increase network throughput.

\section{5 Network Residual Energy}

Residual Energy is one of the most important Metric elements in WSN Routing Optimization because the main goal is to minimize nodes' energy consumption.

Fig. 18 shows average of WSN residual energy with a different number of rounds from (0-2800 rounds) for three compared protocols. Fig. 18, show that the proposed GWOTSA increase Network Residual energy over the compared GA-TSA \&GWO-CSA protocols.

Fig. 19 shows the Average of WSN residual energy with a different number of sensors nodes Start with 100 sensors until 1000 sensors node to show energy consumption for three compared protocols. Fig. 19, show that the proposed GWOTSA enhances network residual energy by $10 \%$ \& $20 \%$ over GA-TSA \& GWO-CSA respectively. GWO-TSA enhances the energy consumption of sensor nodes by using the Tabu search technique for optimal route selection to reduce energy consumption that leads to increased residual energy of the network.

Table II explain the Performance of the proposed GWOTSA over the compared protocols GA-TSA \& GWO-CSA. The experimental results indicate that the proposed GWO-TSA enhances WSN Energy consumption by $10 \%$ and $20 \%$, Increase in WSN Lifetime by $13 \%$ and $18 \%$ and finally Increase in WSN Throughput by $6 \%$ and $14 \%$ over GA-TSA and GWO-TSA respectively.

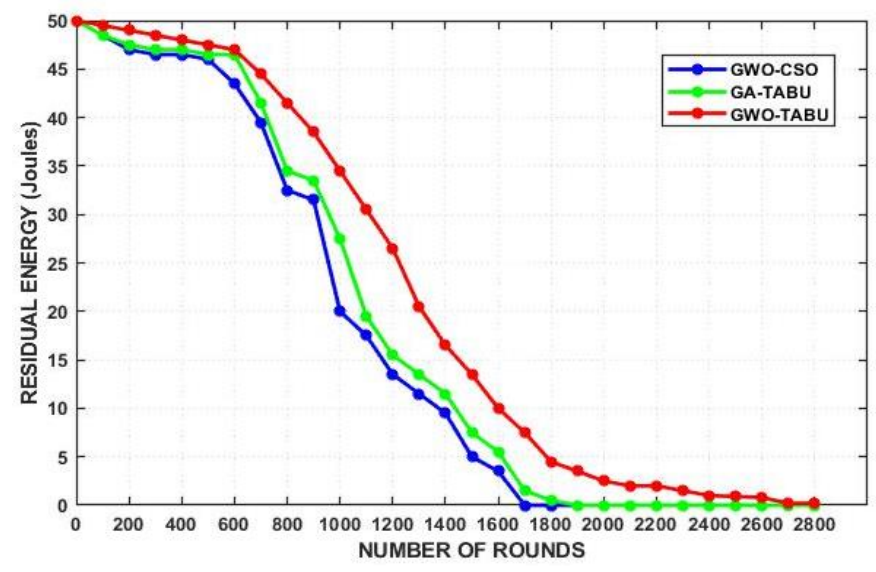

Fig. 18. Network Residual Energy with a different Number of Rounds.

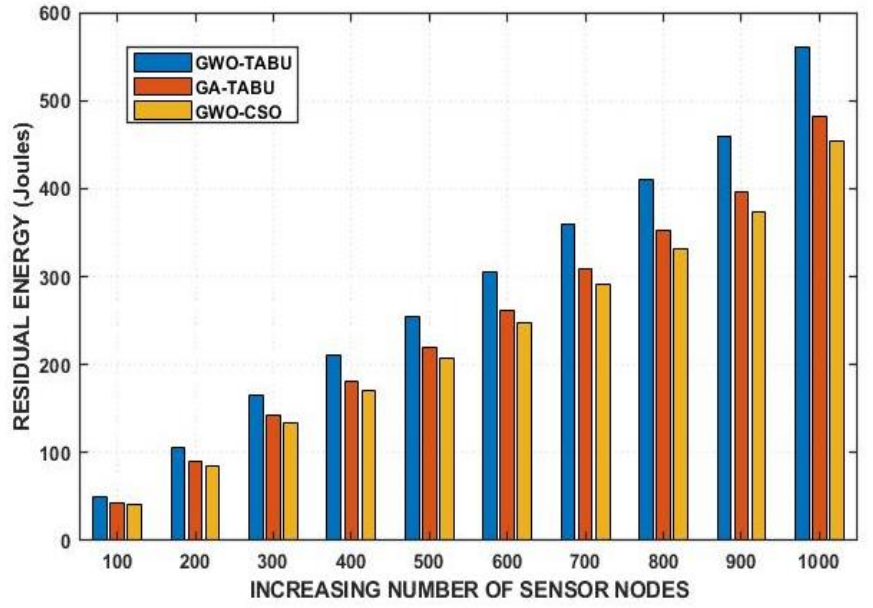

Fig. 19. Network Residaul Energy of a different Number of Sensor Nodes.

TABLE II. PROPOSED GWO-TSA PERFORMANCE

\begin{tabular}{|l|l|l|l|}
\hline \multirow{2}{*}{ protocols } & \multicolumn{3}{|l|}{ WSN Performance } \\
\cline { 2 - 4 } & Energy consumption & Lifetime & Throughput \\
\hline GA-TSA & $10 \%$ & $13 \%$ & $6 \%$ \\
\hline GWO-CSA & $20 \%$ & $18 \%$ & $14 \%$ \\
\hline
\end{tabular}

\section{VII.CONCLUSION}

In this paper, we propose a new energy-efficient multi-hop routing protocol based on GWO and TSA, with the primary goal of optimizing routing by reducing the energy consumption of Sensor nodes, extending the lifetime of WSNs, and increasing network throughput. The proposed GWO-TSA protocol accomplished this goal by incorporating GWO into the $\mathrm{CH}$ selection process. By utilizing a fitness function based on the sensor node's energy residual and its distance to BS, TSA determined the optimal route from the $\mathrm{CH}$ to BS using FRRPs. The suggested GWO-TSA satisfies QoS requirements such as network scalability, since the proposed GWO- TSA's performance is unaffected by the number of sensor nodes but is influenced by the node's energy and distance to the base station, and network dependability. TSA identifies several optimal routes for data transmission from $\mathrm{CH}$ to $\mathrm{BS}$ to increase data transmission reliability; rather than using a single path, many paths may be utilized concurrently. Through simulations in MATLAB, it was shown that the proposed approach accomplished the route optimization goal by combining GWO and TSA.

\section{ACKNOWLEDGMENT}

I express my sincere gratitude and special thanks to my guides Assoc.Prof. Rowayda A.Sadek \& Mohamed M.Abbassy for their constant help, encouragement and inspiration throughout the thesis work. Without their valuable guidance, this work would never have been a successful one. I would also like to thank my parents and my husband who have been the backbone, advisors and constant source of motivation throughout the work. 


\section{REFERENCES}

[1] P. Srinivasa Ragavan and - K. Ramasamy, Optimized routing in wireless sensor networks by establishing dynamic topologies based on genetic algorithm, Springer,2019.

[2] K. Johny Elma and Dr.S .Meenakshi, Dynamic cluster formation method to increase the lifetime of a wireless sensor network, International Journal of Computer Science and Information Security (IJCSIS) ,2017.

[3] M. Kumar Singh, S. Intekhab Amin, S. Akhtar Imam , V. Kumar Sachan and A. Choudhary, A survey of wireless sensor network and its types, International Conference on Advances in Computing, Communication Control and Networking, 2018 .

[4] A. Kau \&, Dr. S. Vatta, Implementation of LEACH, Hetero-LEACH, SEP and EEHC protocols using MATLAB in wireless sensor network, International Journal of Engineering Research \& Technology (IJERT) ,2016.

[5] H. Chaurasiya \&Dr.S. Ghosh, Performance evaluation of energyefficient cluster-based algorithms in wireless sensor network, International Journal of Advanced Trends in Computer Science and Engineering, 2018.

[6] X. Zhao, H. Zhu, S. Aleksic and Q. Gao, Energy-efficient routing protocol for wireless sensor networks based on improved grey wolf optimizer, KSII Transactions on Internet and Information Systems, 2018.

[7] X. Zhao , S. Ren ,H. Quan and Q. Gao , Routing protocol for heterogeneous wireless sensor networks based on a modified grey wolf optimizer, Sensors ,2020.

[8] S. Al-Khammasi, D. Alhelal and N. Salih Ali, Energy efficient cluster based routing protocol for dynamic and static nodes in wireless sensor network, TELKOMNIKA .2018.

[9] Y R. Sadek, G. Selim and T. Abdel-Hakam, A novel energy efficient vice cluster head routing protocol in wireless sensor networks, IEEE Seventh International Conference on Intelligent Computing and Information Systems (ICICIS), 2015.

[10] N. A. Al-Aboody \& H. S. Al-Raweshidy, Grey wolf optimization-based energy-efficient routing protocol for heterogeneous wireless sensor networks", IEEE, 2016.

[11] A. Rai, and M. Sharma, Wireless sensor network using tabu searching algorithm and fuzzy inference system, International Journal of Recent Technology and Engineering (IJRTE) ,2019.

[12] P. Subramanian, J. Martin Sahayaraj, S. Senthilkumar and D. Stalin Alex, A hybrid grey wolf and crow search optimization algorithm-based optimal cluster head selection scheme for wireless sensor network.", Springer, 2020.

[13] Z. Ming Gao and J. Zhao, An improved grey wolf optimization algorithm with variable weights", Hindawi Computational Intelligence and Neuroscience ,2019.

[14] P. HU, S. CHEN, H. HUANG, G. ZHANG, and L. LIU, " Improved alpha-guided grey wolf optimizer", IEEE Access ,2018.

[15] M. Sharawi \& E. Emary, " Impact of grey wolf optimization on wsn cluster formation and lifetime expansion", IEEE ,2017.

[16] A. Rathee, R. Singh \& A. Nandini, Wireless sensor network- challenges and possibilities, International Journal of Computer Applications, 2016.

[17] M. Akhtar, A. Ali , Z. Ali , M. Hashmi and M. Atif, Cluster based routing protocols for Wireless Sensor Networks: An Overview", (IJACSA) International Journal of Advanced Computer Science and Applications ,2018.

[18] H. Faris I. Aljarah, M. Al-Betar, and S. Mirjalili, Grey wolf optimizer: a review of recent variants and applications", Neural Computing and Applications ,2018.

[19] A. Yadav and S. Kumar, Energy efficient cluster formation in wireless sensor networks using particle swarm optimization, Indian Journal of Science and Technology,2017.

[20] W. Long \& S. Xu ," A novel grey wolf optimizer for global optimization problems, IEEE ,2016.

[21] H. Orojloo \& A. Haghighat, A tabu search based routing algorithm for wireless sensor Networks, Springer , 2016.

[22] M. Singh Manshahia: Wireless sensor networks: a survey ", International Journal of Scientific \& Engineering Research, 2016.

[23] H. M. Salman, Survey of routing protocols in wireless sensor networks, nternational Journal of Sensors and Sensor Networks,2014.

[24] A. Sabri , K. Al-Shqeerat, Hierarchical cluster-based routing protocols for wireless sensor networks - a survey, IJCSI International Journal of Computer Science Issues,2014.

[25] A. Singh, Dr. S.B. Rana, Heterogeneous routing protocols in wireless sensor network: a survey, International Research Journal of Engineering and Technology (IRJET),2015.

[26] S.P. Singh,S.C. Sharma, A survey on cluster based routing protocols in wireless sensor networks, International Conference on Advanced Computing Technologies and Applications (ICACTA),2015. 